\title{
Effects of thermal conduction and compressive viscosity on the period ratio of the slow mode
}

\author{
C. K. Macnamara and B. Roberts
}

\begin{abstract}
School of Mathematics and Statistics, University of St. Andrews, St. Andrews, Fife, KY16 9SS, Scotland
e-mail: cicely@mcs.st-andrews.ac.uk
\end{abstract}

Received 5 October 2009 / Accepted 8 March 2010

\section{ABSTRACT}

\begin{abstract}
Aims. Increasing observational evidence of wave modes brings us to a closer understanding of the solar corona. Coronal seismology allows us to combine wave observations and theory to determine otherwise unknown parameters. The period ratio, $P_{1} / 2 P_{2}$, between the period $P_{1}$ of the fundamental mode and the period $P_{2}$ of its first overtone, is one such tool of coronal seismology and its departure from unity provides information about the structure of the corona.

Methods. We consider analytically the effects of thermal conduction and compressive viscosity on the period ratio for a longitudinally propagating sound wave.

Results. For coronal values of thermal conduction the effect on the period ratio is negligible. For compressive viscosity the effect on the period ratio may become important for some short hot loops.

Conclusions. Damping typically has a small effect on the period ratio, suggesting that longitudinal structuring remains the most significant effect.
\end{abstract}

Key words. Sun: corona - Sun: oscillations

\section{Introduction}

Since the late 1990s, Solar and Heliospheric Observatory (SoHO) and Transition Region and Corona Explorer (TRACE) observations of various coronal wave phenomena have been possible (see, for example, Nakariakov \& Verwichte 2005). This has led to an increased growth in the field of coronal seismology of magnetic loops suggested 25 years ago (Roberts et al. 1984). Recent observations with Hinode and STEREO have added further to this record (see, for example, Wang et al. 2009; Marsh et al. 2009). Coronal seismology helps to unveil the nature of the solar atmosphere; studying observed waves and drawing on their specific properties, it is possible to diagnose aspects of the coronal structure which might otherwise remain unknown. The period ratio between the fundamental mode and its first overtone has been noted as an effective tool for coronal seismology (Andries et al. 2005a,b, 2009; Goossens et al. 2006; McEwan et al. 2006, 2008; Donnelly et al. 2006; Dymova \& Ruderman 2007; Díaz et al. 2007; Roberts 2008; Verth \& Erdélyi 2008; Ruderman et al. 2008; Erdélyi \& Morton 2009; Morton \& Erdélyi 2009). This topic has recently been reviewed in Andries et al. (2009).

There is ever increasing observational evidence of magnetoacoustic waves, both standing and propagating, occurring in the corona. Propagating slow waves have been reported by Ofman et al. (1997, 1999), DeForest \& Gurman (1998), De Moortel et al. (2000, 2002a,b), Robbrecht et al. (2001), Ofman \& Wang (2002), McEwan \& De Moortel (2006) and Marsh et al. (2009). Standing slow waves have been observed by Wang et al. (2002, 2003, 2009) and Srivastava \& Dwivedi (2010). Standing fast waves in the form of transverse kink waves have been recorded by Aschwanden et al. (1999, 2002), Nakariakov et al. (1999), Wang \& Solanki (2004), Verwichte et al. (2004) and Van Doorsselaere et al. (2007), while standing fast sausage modes have been observed by Nakariakov et al. (2003) and Melnikov et al. (2005), and modelled by Pascoe et al. (2007, 2009), Srivastava et al. (2008) and Inglis et al. (2009).

Standing slow modes are of particular interest here. They have been extensively studied by Wang et al. $(2002,2003,2009)$ using SoHO/SUMER observations and in this form are recorded in hot loops only. However, very recently intensity oscillations in the cooler loops observed by the EUV imaging spectrometer (EIS) onboard the Hinode spacecraft have been reported by Srivastava \& Dwivedi (2010) and are interpreted as the signature of slow acoustic oscillations in a non-flaring loop.

Our interest is in the detection of multi-periods in loops. Multi-periods (typically the fundamental mode and its first overtone) were first reported in standing fast waves (Verwichte et al. 2004; Van Doorsselaere et al. 2007; De Moortel \& Brady 2007; O'Shea et al. 2007; Srivastava et al. 2008) and until very recently had not been found in slow modes. In fast waves the observed tendency for the period ratio $P_{1} / 2 P_{2}$ between the fundamental mode of period $P_{1}$ and the period $P_{2}$ of its first overtone to be less than unity (the value for a simple wave on a string) has led to an interest in this ratio. A large number of physical effects have been assessed for their influence on the period ratio: wave dispersion, gravitational stratification, longitudinal and transverse density structuring, loop cross-sectional ellipticity, the overall geometry of a loop and magnetic field expansion (Andries et al. 2005a,b; McEwan et al. 2006, 2008; Díaz et al. 2007; Ruderman et al. 2008; Verth \& Erdélyi 2008; Erdélyi \& Morton 2009; Morton \& Erdélyi 2009; Inglis et al. 2009). The overall conclusion seems to be that longitudinal structuring plays the most marked role (Andries et al. 2009). Longitudinal structuring may take the form of density stratification (e.g. Andries et al. 2005a,b; McEwan et al. 2006, 2008) or magnetic structuring 
(Verth \& Erdélyi 2008). The recent reports by Srivastava \& Dwivedi (2010) suggest that slow modes also form multiperiods; interestingly, their reported period ratios of $P_{1} / P_{2}=$ 1.54 and 1.84 (corresponding to $P_{1} / 2 P_{2}=0.77$ and 0.92 ) show a strong departure from canonical values, comparable or even larger than the period ratios measured in fast waves.

The effect of damping on the period ratio has not been assessed. We take up this topic here. We discuss explicitly the onedimensional sound wave and the role of thermal conduction and compressive viscosity on determining the period ratio. This case is directly relevant to the propagation of the slow magnetoacoustic wave under coronal conditions, since for a low $\beta$ plasma the slow mode has been shown to decouple from the magnetohydrodynamic equations and obey a Klein-Gordon equation (Roberts 2006); in the absence of stratification, the Klein-Gordon equation reduces to the one-dimensional wave equation.

De Moortel \& Hood (2003) studied generally the effect of both thermal conduction and compressive viscosity, as well as gravitational stratification and magnetic field divergence, on the slow mode for TRACE loops (1-2 MK temperatures), and were able to conclude that thermal conduction had an important role. On the other hand, Sigalotti et al. (2007) have argued that compressive viscosity plays a significant role when we consider hot ( $\geq 5 \mathrm{MK}$ ) loops, as observed by SoHO/SUMER.

Here we consider the combined effects of thermal conduction and compressive viscosity on the period ratio, $P_{1} / 2 P_{2}$, of a sound wave propagating one-dimensionally. This is the first discussion of the influence of non-ideal effects on period ratios. We obtain the dispersion relation for sound waves influenced by thermal conduction and compressive viscosity and use this to determine $P_{1} / 2 P_{2}$. This raises the possibility of using the period ratio as a diagnostic tool of non-ideal conditions.

\section{Period ratio for slow modes}

We model a single coronal loop line-tied at footpoints located in the photosphere. Gravity and field-line curvature are neglected. We treat the longitudinally propagating waves as purely one dimensional sound waves. It is generally considered that the plasma- $\beta$ is small in the corona (see, for example, the discussion in Ruderman \& Roberts 2002), allowing us to use the onedimensional form of the acoustic equations (Roberts 2006).

Consider, then, the acoustic equations

$$
\begin{aligned}
& \frac{\mathrm{D} \rho}{\mathrm{D} t}+\rho \nabla \cdot \boldsymbol{v}=0, \\
& \rho \frac{\mathrm{D} \boldsymbol{v}}{\mathrm{D} t}+\nabla p=\boldsymbol{F}_{\nu}, \\
& \frac{\mathrm{D} p}{\mathrm{D} t}-\frac{\gamma p}{\rho} \frac{\mathrm{D} \rho}{\mathrm{D} t}-(\gamma-1) \nabla \cdot\left(\kappa_{\|} \nabla T\right)=0, \\
& p-\frac{R}{\tilde{\mu}} \rho T=0 .
\end{aligned}
$$

Here $\rho p, v$ and $T$ represent the density, pressure, velocity and temperature respectively; $R$ is the gas constant, $\tilde{\mu}$ is the mean molecular weight and $\gamma$ is the ratio of specific heats (taken to be $\gamma=5 / 3$ ). The thermal conductivity is $\kappa_{\|}$and $\boldsymbol{F}_{v}$ represents the viscous forces. The operator $D / D t=\partial / \partial t+\boldsymbol{v} \cdot \nabla$ is the material derivative. Thermal conduction is strongly suppressed across a magnetic field (Spitzer 1962) and so we take thermal conduction to act purely along the $z$-axis, setting $\kappa_{\|}=\kappa_{0} T^{5 / 2} \mathrm{~W} \mathrm{~m}^{-1} \mathrm{deg}^{-1}$ with $\kappa_{0}=10^{-11}$ in mks units (De Moortel \& Hood 2003). Viscous forces are here represented by the compressive viscosity taken to act purely along the $z$-axis (with unit vector $\hat{z}$ ), so that (see Ofman et al. 1994; Ofman \& Wang 2002; De Moortel \& Hood 2003; Mendoza-Briceño et al. 2004; Sigalotti et al. 2007)

$$
\boldsymbol{F}_{v}=\frac{4}{3} \frac{\partial}{\partial z}\left(v \frac{\partial v_{z}}{\partial z}\right) \hat{z}
$$

where the coefficient of viscosity $v$ is of the form $v=$ $v_{0} T^{5 / 2} \mathrm{~kg} \mathrm{~m}^{-1} \mathrm{~s}^{-1}$ with $v_{0}=10^{-17}$ (Hollweg 1985).

Equations (1)-(4) are linearised with respect to small perturbations about a uniform equilibrium with density $\rho_{0}$, pressure $p_{0}$ and temperature $T_{0}$. The velocity perturbation $\boldsymbol{v}=v_{z} \hat{z}$ is taken to be purely longitudinal (along the direction of the imposed magnetic field for a slow mode). The linear equations are

$$
\begin{aligned}
& \frac{\partial \rho}{\partial t}+\rho_{0} \frac{\partial v_{z}}{\partial z}=0, \\
& \rho_{0} \frac{\partial v_{z}}{\partial t}+\frac{\partial p}{\partial z}=\frac{4}{3} v \frac{\partial^{2} v_{z}}{\partial z^{2}}, \\
& \frac{\partial p}{\partial t}-\frac{\gamma p_{0}}{\rho_{0}} \frac{\partial \rho}{\partial t}-(\gamma-1) \kappa_{\|} \frac{\partial^{2} T}{\partial z^{2}}=0, \\
& \frac{p}{p_{0}}-\frac{\rho}{\rho_{0}}-\frac{T}{T_{0}}=0,
\end{aligned}
$$

where now $v=v_{0} T_{0}^{5 / 2}$ and $\kappa_{\|}=\kappa_{0} T_{0}^{5 / 2}$.

The linearised Eqs. (6)-(9) lead to the wave-like equation

$$
\begin{aligned}
& \left(\frac{\partial}{\partial t}-(\gamma-1) \frac{\kappa_{\|} T_{0}}{\gamma p_{0}} \frac{\partial^{2}}{\partial z^{2}}+\frac{4}{3} \frac{v}{\gamma p_{0}} \frac{\partial^{2}}{\partial t^{2}}\right. \\
& \left.-\frac{4}{3} \frac{v}{\gamma p_{0}} \gamma(\gamma-1) \frac{\kappa_{\|} T_{0}}{\gamma p_{0}} \frac{\partial^{3}}{\partial t \partial z^{2}}\right) c_{\mathrm{s}}^{2} \frac{\partial^{2} v_{z}}{\partial z^{2}} \\
& =\left(\frac{\partial}{\partial t}-\gamma(\gamma-1) \frac{\kappa_{\|} T_{0}}{\gamma p_{0}} \frac{\partial^{2}}{\partial z^{2}}\right) \frac{\partial^{2} v_{z}}{\partial t^{2}},
\end{aligned}
$$

where $c_{\mathrm{s}}^{2}=\gamma p_{0} / \rho_{0}$ is the square of the sound speed.

Considering Fourier analysis of Eq. (10), we write

$$
v_{z}(z, t)=v_{z 0} \mathrm{e}^{\mathrm{i}\left(\omega t-k_{z} z\right)}
$$

for frequency $\omega$, wavenumber $k_{z}$ and complex amplitude $v_{z 0}$. The dispersion relation for sound waves under the combined effects of thermal conduction $\kappa_{\|}$and compressive viscosity $v$ is then obtained,

$$
\begin{aligned}
& \omega^{3}-\mathrm{i}\left(\frac{4}{3} \frac{v}{\gamma p_{0}} c_{\mathrm{s}}^{2}+\gamma \frac{(\gamma-1) \kappa_{\|} T_{0}}{\gamma p_{0}}\right) k_{z}^{2} \omega^{2} \\
& -\left(c_{\mathrm{s}}^{2}+\frac{4}{3} \frac{v}{\gamma p_{0}} c_{\mathrm{s}}^{2} \gamma \frac{(\gamma-1) \kappa_{\|} T_{0}}{\gamma p_{0}} k_{z}^{2}\right) k_{z}^{2} \omega \\
& +\mathrm{i} \frac{(\gamma-1) \kappa_{\|} T_{0}}{\gamma p_{0}} c_{\mathrm{s}}^{2} k_{z}^{4}=0 .
\end{aligned}
$$

To model a coronal loop, we suppose that it has a length $2 L$ with its apex at $z=0$ and its footpoints at $z= \pm L$. For motions $v_{z}$ that vanish at the footpoints but nowhere else within $-L<z<L$, we take $k_{z}=\pi / 2 L$ (with corresponding wavelength $2 \pi / k_{z}=4 L$, or twice the loop length); this is the fundamental mode of oscillation. The first overtone of the fundamental has a wavelength that is half the wavelength of the fundamental, corresponding to $k_{z}=\pi / L ; v_{z}$ now vanishes at the loop apex $(z=0)$ as well as at the footpoints.

The dispersion relation (12) determines the (complex) values of $\omega$ and may be used to determine the period ratio $P_{1} / 2 P_{2}$. 
Writing $\omega=\omega_{\mathrm{r}}+\mathrm{i} \omega_{\mathrm{i}}$, where $\omega_{\mathrm{r}}$ is the real part of $\omega$ and $\omega_{\mathrm{i}}$ denotes the imaginary part, we may solve (12) with $k_{z} L=\pi / 2$ to obtain $\omega_{1}=\operatorname{Real}(\omega)$, the value of $\omega_{\mathrm{r}}$ for the fundamental mode. Similarly, we may solve (12) with $k_{z} L=\pi$ to obtain $\omega_{2}=\operatorname{Real}(\omega)$, the value of $\omega_{r}$ for the first overtone. Then, since the fundamental period is $P_{1}=2 \pi / \omega_{1}$ and the first overtone has the period $P_{2}=2 \pi / \omega_{2}$, we have

$\frac{P_{1}}{2 P_{2}}=\frac{\omega_{2}}{2 \omega_{1}}$.

It is convenient to introduce the non-dimensional constants

$\epsilon=\frac{4}{3} v \frac{c_{\mathrm{S}}}{\gamma p_{0} L}=\frac{4}{3} v_{0} T_{0}^{3} \frac{\sqrt{\frac{\gamma R}{\tilde{\mu}}}}{\gamma p_{0} L}$,

$d=\frac{1}{\gamma} \frac{\tau_{\mathrm{s}}}{\tau_{\text {cond }}}=\frac{(\gamma-1) \kappa_{\|} T_{0}}{\gamma p_{0} L c_{\mathrm{s}}}=\frac{(\gamma-1) \kappa_{0} T_{0}^{3}}{\gamma p_{0} L \sqrt{\frac{\gamma R}{\tilde{\mu}}}}$

where $\tau_{\mathrm{s}}=L / c_{\mathrm{S}}$ is the sound travel time and $\tau_{\text {cond }}=L^{2} p_{0} /(\gamma-$ 1) $\kappa_{\|} T_{0}$ is the thermal conduction timescale for a loop of halflength $L$. Then, setting $\Omega=\omega / k_{z} c_{\mathrm{s}}$ the dispersion relation (12) becomes

$\Omega^{3}-\mathrm{i}(\mathcal{V}+\gamma \mathcal{D}) \Omega^{2}-(1+\gamma \mathcal{V} \mathcal{D}) \Omega+\mathrm{i} \mathcal{D}=0$,

where

$\mathcal{V}=\epsilon k_{z} L, \quad \mathcal{D}=d k_{z} L$.

It is noted that both $\mathcal{V}$ and $\mathcal{D}$ have a dependence upon temperature, pressure and loop half-length that is of the same form:

$\mathcal{V}=\mathcal{V}_{0} \frac{T_{0}^{3}}{p_{0} L}, \quad \mathcal{D}=\mathcal{D}_{0} \frac{T_{0}^{3}}{p_{0} L}$,

where $\mathcal{V}_{0}$ and $\mathcal{D}_{0}$ are constants. The values of $\mathcal{V}$ and $\mathcal{D}$ tend to zero in the limit of low temperatures, high pressures or long loop lengths.

With $\epsilon=d=0$ (i.e, in the absence of thermal conduction and compressive viscosity), $\Omega=0$ or $\Omega= \pm 1$ and so $\omega=k_{z} c_{\mathrm{S}}$ provides a solution of (12). The frequency $\omega_{1}$ of the fundamental mode is $\omega_{1}=\pi c_{\mathrm{s}} /(2 L)$ and the frequency $\omega_{2}$ of the first overtone is $\pi c_{\mathrm{s}} / L$. Thus the period ratio $P_{1} / 2 P_{2}$ formed from the fundamental period $P_{1}\left(=2 \pi / \omega_{1}\right)$ and the period $P_{2}\left(=2 \pi / \omega_{2}\right)$ of the first overtone is unity, when $\epsilon=d=0$. It is departures of $P_{1} / 2 P_{2}$ from unity that are of interest. Here we consider how thermal conduction $\kappa_{\|}$and viscosity $v$ bring about shifts in the period ratio from unity.

\subsection{The effect of thermal conduction}

In the absence of compressive viscosity $(v=0)$ Eq. (10) reduces to

$$
\begin{aligned}
& \left(\frac{\partial}{\partial t}-(\gamma-1) \frac{\kappa_{\|} T_{0}}{\gamma p_{0}} \frac{\partial^{2}}{\partial z^{2}}\right) c_{\mathrm{s}}^{2} \frac{\partial^{2} v_{z}}{\partial z^{2}} \\
& =\left(\frac{\partial}{\partial t}-\gamma(\gamma-1) \frac{\kappa_{\|} T_{0}}{\gamma p_{0}} \frac{\partial^{2}}{\partial z^{2}}\right) \frac{\partial^{2} v_{z}}{\partial t^{2}} .
\end{aligned}
$$

The associated dispersion relation follows from (15) with $\mathcal{V}=0$ :

$\Omega^{3}-\mathrm{i} \gamma \mathcal{D} \Omega^{2}-\Omega+\mathrm{i} \mathcal{D}=0$.

A relation of this form has been obtained by Field (1965). This dispersion relation is in the form of a cubic which can be solved using the Cardano method with Vièta substitution (Abramowitz \& Stegun 1965; Press et al. 1986) to give

$\Omega=\frac{1}{6}\left(\mathrm{i} C_{1} \pm 12 \sqrt{C_{2}}\right)^{1 / 3}+\frac{2 C_{3}}{\left(\mathrm{i} C_{1} \pm 12 \sqrt{C_{2}}\right)^{1 / 3}}+\mathrm{i} C_{4}$

where

$C_{1}=(36 \gamma-108) \mathcal{D}-8 \gamma^{3} \mathcal{D}^{3}$,

$C_{2}=-12+\left(54 \gamma+3 \gamma^{2}-81\right) \mathcal{D}^{2}-12 \gamma^{3} \mathcal{D}^{4}$,

$C_{3}=1-\frac{1}{3} \gamma^{2} \mathcal{D}^{2}, \quad C_{4}=\frac{1}{3} \gamma \mathcal{D}$.

We choose the root $(20)$ such that real $(\Omega)>0$.

Note that in the absence of thermal conduction $(\mathcal{D}=0)$, $C_{1}=C_{4}=0, C_{2}=-12, C_{3}=1$ and Eq. (20) leads to three solutions for $\Omega$, namely $\Omega=0$ or $\Omega^{2}=1$. Also, for $D \gg 1$ the dispersion relation (19) gives roots $\Omega=0$ and $\Omega^{2}=1 / \gamma$. Thus in either extreme of weak thermal conduction $(\mathcal{D} \ll 1)$ or strong thermal conduction $(\mathcal{D} \gg 1)$ sound propagates without damping, at the sound speed $c_{\mathrm{s}}$ for $\mathcal{D}=0$ and at the Newtonian sound speed $c_{\mathrm{s}} / \sqrt{\gamma}\left(=\left(p_{0} / \rho_{0}\right)^{1 / 2}\right)$ for $\mathcal{D} \rightarrow \infty$. The absence of any damping $(\operatorname{imag}(\Omega) \equiv 0)$ in these two extremes makes clear that there is an intermediate value of $\mathcal{D}$ for which damping is a maximum. Finally, we note that if $\gamma=1$ then the dispersion relation (19) has the general solutions $\Omega=\mathcal{D}$ and $\Omega^{2}=1$, corresponding to the thermal mode and the isothermal propagation of sound at the Newtonian sound speed.

Thus, the importance (or otherwise) of thermal conduction depends entirely on the magnitude of the dimensionless parameter $\mathcal{D}$, which in turn depends on the loop half-length $L$ entirely through the wavenumber $k_{z}$ (the product $d L$ being independent of $L)$. The parameter $\mathcal{D}\left(=d k_{z} L\right)$ thus depends upon the magnitude of the thermal coefficient $d$, with $k_{z} L=\pi / 2$ for the fundamental mode and $k_{z} L=\pi$ for the first overtone. Following Sigalotti et al. (2007), we give in Table 1 the values of $d$ for various loop temperatures $T_{0}$ and loop lengths $2 L$; we fix the equilibrium loop pressure $p_{0}$, choosing $p_{0}=0.055 \mathrm{~Pa}$ $\left(=0.55\right.$ dynes $\left.\mathrm{cm}^{-2}\right)$. We take the ratio of specific heats $\gamma$ to be $5 / 3$, the gas constant $R=8.3 \times 10^{3} \mathrm{~m}^{2} \mathrm{~s}^{-2} \mathrm{deg}^{-1}$, the mean molecular weight $\tilde{\mu}=0.6$, and the thermal conduction coefficient $\kappa_{0}=10^{-11}$ in mks units. With fixed loop pressure $p_{0}$, the thermal measure $d$ varies with the cube of the loop temperature and inversely with the loop half-length $L: d \propto T_{0}^{3} / L$. As an illustration, a typical TRACE loop with temperature $T_{0}=1 \mathrm{MK}$ and a loop length $100 \mathrm{Mm}(L=50 \mathrm{Mm})$ gives $d=0.0096$, and thus $\mathcal{D} \approx 0.015$ for the fundamental mode and $\mathcal{D} \approx 0.030$ for its first overtone. On the other hand, for a typical SUMER hot loop with temperature $T_{0}=6 \mathrm{MK}$ and length $2 L=100 \mathrm{Mm}$ we obtain $d=2.0692$, and $\mathcal{D} \approx 3.25$ for the fundamental mode and $\mathcal{D} \approx 6.50$ for its first overtone. Table 1 illustrates the range of values that arise. In general, TRACE loops have $\mathcal{D} \ll 1$ and SUMER loops have $\mathcal{D} \gg 1$.

The period ratio can be calculated from Eq. (13) where $\omega_{1}=$ $\Omega_{1} k_{z} c_{\mathrm{s}}, \Omega_{1}$ corresponding to the solution in Eq. (20) with $\mathcal{D}=$ $d \pi / 2$ and $\omega_{2}=\Omega_{2} k_{z} c_{\mathrm{s}}, \Omega_{2}$ being the solution corresponding to $\mathcal{D}=d \pi$. Thus, the period ratio may be found from (20); the results are displayed in Fig. 1.

Figure 1 shows the behaviour of the period ratio with $d$ for the exact solution given by Eq. (20). When $d=0$ the period ratio is unity, it then decreases from unity until a particular $d$ value for which the period ratio has a minimum; numerically, the minimum value of the period ratio is found to be $P_{1} / 2 P_{2}=0.897$ occurring at $d=0.291$. The period ratio then increases and for $d$ sufficiently large it returns to unity. 
Table 1. Values of the thermal conduction parameter $d$.

\begin{tabular}{cccccc}
\hline \hline$T_{0}(\mathrm{MK})$ & $d(2 L=50 \mathrm{Mm})$ & $d(2 L=100 \mathrm{Mm})$ & $d(2 L=200 \mathrm{Mm})$ & $d(2 L=300 \mathrm{Mm})$ & $d(2 L=400 \mathrm{Mm})$ \\
\hline 1 & 0.0192 & 0.0096 & 0.0048 & 0.0032 & 0.0024 \\
2 & 0.1533 & 0.0766 & 0.0383 & 0.0255 & 0.0192 \\
3 & 0.5173 & 0.2586 & 0.1293 & 0.0862 & 0.0647 \\
4 & 1.2262 & 0.6131 & 0.3065 & 0.2044 & 0.1533 \\
5 & 2.3949 & 1.1974 & 0.5987 & 0.3991 & 0.2994 \\
6 & 4.1383 & 2.0692 & 1.0346 & 0.6897 & 0.5173 \\
7 & 6.5715 & 3.2857 & 1.6429 & 1.0953 & 0.8214 \\
8 & 9.8094 & 4.9047 & 2.4523 & 1.6349 & 1.2262 \\
9 & 13.9668 & 6.9834 & 3.4917 & 2.3278 & 1.7459 \\
10 & 19.1589 & 9.5795 & 4.7897 & 3.1932 & 2.3949 \\
\hline
\end{tabular}

Notes. Variations in the thermal conduction parameter $d$ with changes in temperature $T_{0}$ for different loop lengths $2 L$. We have set the equilibrium pressure $p_{0}=0.055 \mathrm{~Pa}$. Also, $\gamma=5 / 3, \tilde{\mu}=0.6$ and $\kappa_{0}=10^{-11}$ in mks units. See also Sigalotti et al. (2007).

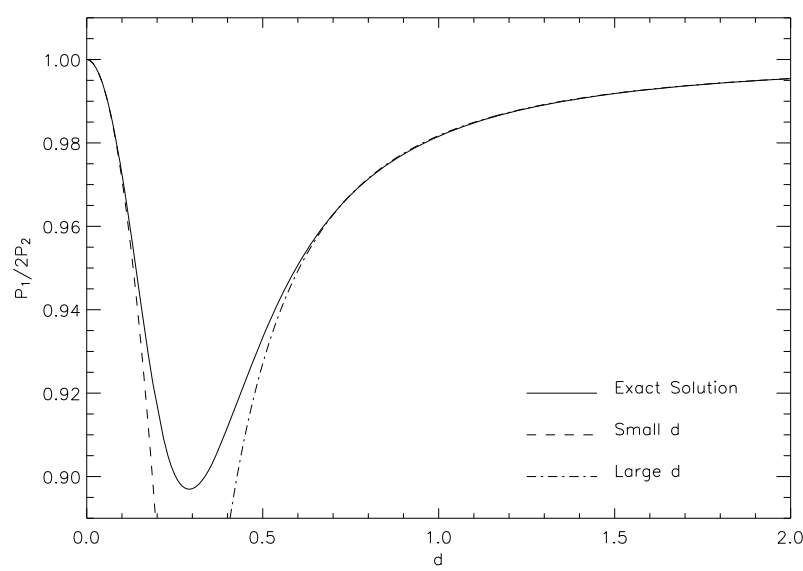

Fig. 1. The period ratio $P_{1} / 2 P_{2}$ as a function of the thermal conduction measure $d$. The solid line indicates the exact solution derived from Eq. (20), the dashed and dot-dashed curves indicate approximations for small and large $d$ as given by Eqs. (27) and (30).

Consulting Table 1 and Fig. 1 we note that for coronal values of $d$ (for both TRACE and SUMER loops) the period ratio is close to unity, suggesting that thermal conduction does not have a dominating effect on the period ratio. However, it appears that for a long $(400 \mathrm{Mm})$ SUMER loop $\left(T_{0}=6 \mathrm{MK}\right)$ with $d \approx 0.517$ it may be possible for the period ratio to reduce to 0.937 (obtained from the exact solution).

It is straightforward to obtain a series expansion for $\Omega$ in powers of $\mathcal{D}$ directly from the dispersion relation (19) in order to approximate the value of the period ratio for small $\mathcal{D}$. The result is

$\Omega=1+\frac{1}{2} \mathrm{i}(\gamma-1) \mathcal{D}-\frac{1}{8}(\gamma-1)(\gamma+3) \mathcal{D}^{2}+\ldots, \quad \mathcal{D} \ll 1$.

Setting $k_{z} L=\pi / 2$ for the fundamental mode then gives

$\omega_{1}=\frac{\pi c_{\mathrm{s}}}{2 L}\left[1-\frac{1}{8}(\gamma-1)(\gamma+3)\left(\frac{d \pi}{2}\right)^{2}+\ldots\right], \quad \frac{1}{2} d \pi \ll 1$,

and setting $k_{z} L=\pi$ for the first overtone gives

$\omega_{2}=\frac{\pi c_{\mathrm{s}}}{L}\left[1-\frac{1}{8}(\gamma-1)(\gamma+3)(d \pi)^{2}+\ldots\right], \quad d \pi \ll 1$.

Altogether, then, the period ratio for small $\mathcal{D}$ (i.e. small $d \pi$ ) is

$\frac{P_{1}}{2 P_{2}}=1-\frac{3}{32}(\gamma-1)(\gamma+3)(d \pi)^{2}+\ldots, \quad d \pi \ll 1 ;$ with $\gamma=5 / 3$ this is

$\frac{P_{1}}{2 P_{2}}=1-\frac{7}{24}(d \pi)^{2}+\ldots, \quad d \pi \ll 1$.

In a similar fashion the behaviour for large $\mathcal{D}$ may be determined directly from (19) by expansion about $\Omega=1 / \sqrt{\gamma}$ in inverse powers of $\mathcal{D}$. The result is

$$
\begin{aligned}
\Omega= & \frac{1}{\sqrt{\gamma}}+\frac{1}{2 \gamma^{2}} \mathrm{i}(\gamma-1) \mathcal{D}^{-1} \\
& -\frac{1}{8 \gamma^{3} \sqrt{\gamma}}(\gamma-1)(\gamma-5) \mathcal{D}^{-2}+\ldots, \quad \mathcal{D} \gg 1 .
\end{aligned}
$$

The period ratio is then found to be

$$
\frac{P_{1}}{2 P_{2}}=1+\frac{3}{8 \gamma^{3}}(\gamma-1)(\gamma-5) \frac{1}{(d \pi)^{2}}+\ldots, \quad d \pi \gg 1
$$

with $\gamma=5 / 3$ this is

$$
\frac{P_{1}}{2 P_{2}}=1-\frac{9}{50} \frac{1}{(d \pi)^{2}}+\ldots, \quad d \pi \gg 1 \text {. }
$$

Both the extremes of small and large $d \pi$ may arise. The case $(d \pi)^{2}$ small is likely to occur for TRACE loops; for example, a loop of length $100 \mathrm{Mm}$ and temperature $1 \mathrm{MK}$ gives $d \approx 0.0096$ and $(d \pi)^{2} \approx 0.00091$. On the other hand, for SUMER loops $(d \pi)^{2}$ may be large; for example, a loop of length $100 \mathrm{Mm}$ and temperature $10 \mathrm{MK}$ gives $d \approx 9.58$ leading to $(d \pi)^{2} \approx 906$ and $1 /(d \gamma \pi)^{2} \approx 0.0001$. It should be noted that the approximations (26) and (29) for the period ratio, applying for small or large $d \pi$, fail to capture the minimum behaviour that is present in the exact solution displayed in Fig. 1.

\subsection{The effect of compressive viscosity}

In the absence of thermal conduction $\left(\kappa_{\|}=0\right)$ Eq. (10) reduces to

$\frac{4}{3} \frac{v}{\rho_{0}} \frac{\partial}{\partial t} \frac{\partial^{2} v_{z}}{\partial z^{2}}=\left(\frac{\partial^{2}}{\partial t^{2}}-c_{\mathrm{s}}^{2} \frac{\partial^{2}}{\partial z^{2}}\right) v_{z}$

and the dispersion relation (15) becomes (ignoring the solution $\Omega=0$ )

$\Omega^{2}-\mathrm{i} \mathcal{V} \Omega-1=0$.

As a simple quadratic this dispersion relation is easily solved to give

$\Omega=\frac{1}{2} V_{i} \pm\left(1-\frac{1}{4} V^{2}\right)^{1 / 2}$ 
Table 2. Values of the compressive viscosity parameter $\epsilon$.

\begin{tabular}{cccccc}
\hline \hline$T_{0}(\mathrm{MK})$ & $\epsilon(2 L=50 \mathrm{Mm})$ & $\epsilon(2 L=100 \mathrm{Mm})$ & $\epsilon(2 L=200 \mathrm{Mm})$ & $\epsilon(2 L=300 \mathrm{Mm})$ & $\epsilon(2 L=400 \mathrm{Mm})$ \\
\hline 1 & $0.883 \mathrm{e}-3$ & $0.442 \mathrm{e}-3$ & $0.221 \mathrm{e}-3$ & $0.147 \mathrm{e}-3$ & $0.110 \mathrm{e}-3$ \\
2 & $0.707 \mathrm{e}-2$ & $0.353 \mathrm{e}-2$ & $0.177 \mathrm{e}-2$ & $0.118 \mathrm{e}-2$ & $0.883 \mathrm{e}-3$ \\
3 & $0.239 \mathrm{e}-1$ & $0.119 \mathrm{e}-1$ & $0.596 \mathrm{e}-2$ & $0.398 \mathrm{e}-2$ & $0.298 \mathrm{e}-2$ \\
4 & $0.565 \mathrm{e}-1$ & $0.283 \mathrm{e}-1$ & $0.141 \mathrm{e}-1$ & $0.942 \mathrm{e}-2$ & $0.707 \mathrm{e}-2$ \\
5 & 0.110 & $0.552 \mathrm{e}-1$ & $0.276 \mathrm{e}-1$ & $0.184 \mathrm{e}-1$ & $0.138 \mathrm{e}-1$ \\
6 & 0.191 & $0.954 \mathrm{e}-1$ & $0.477 \mathrm{e}-1$ & $0.318 \mathrm{e}-1$ & $0.239 \mathrm{e}-1$ \\
7 & 0.303 & 0.152 & $0.758 \mathrm{e}-1$ & $0.505 \mathrm{e}-1$ & $0.379 \mathrm{e}-1$ \\
8 & 0.452 & 0.226 & 0.113 & $0.754 \mathrm{e}-1$ & $0.565 \mathrm{e}-1$ \\
9 & 0.644 & 0.322 & 0.161 & 0.107 & $0.805 \mathrm{e}-1$ \\
10 & 0.883 & 0.442 & 0.221 & 0.147 & 0.110 \\
\hline
\end{tabular}

Notes. Variations in the compressive viscosity parameter $\epsilon$ with changes in temperature $T_{0}$ for different loop lengths $2 L$. Again the loop pressure is $p_{0}=0.55 \mathrm{~Pa}$ and the other parameters are as in Table 1 . The compressive viscosity is $v_{0}=10^{-17} \mathrm{in}$ mks units.

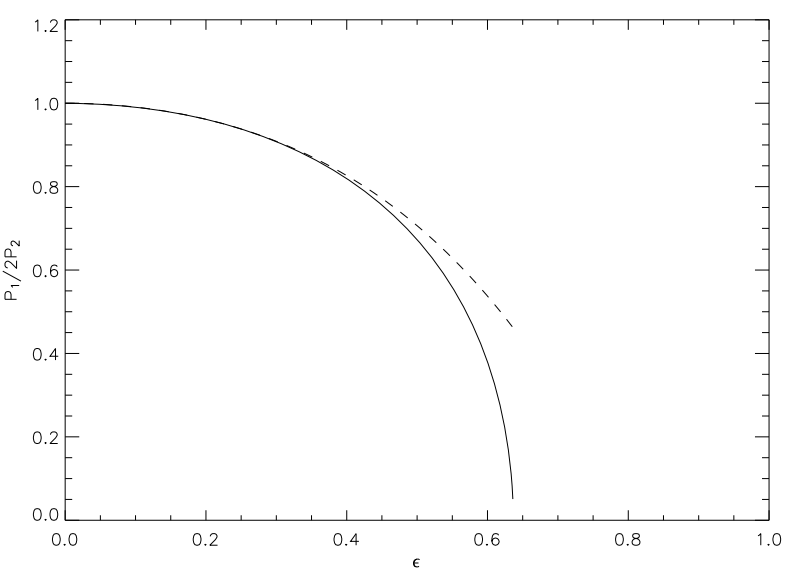

Fig. 2. The period ratio $P_{1} / 2 P_{2}$ as a function of the compressive viscosity measure $\epsilon$. The exact value determined by the dispersion relation (32) is shown as a full curve, with the approximation (35) shown dashed.

In order to obtain a wave period we require that $\mathcal{V}<2$, so $\epsilon$ is constrained by $\epsilon<2 / k_{z} L$ or else the motion is purely damped. Since $k_{z}=\pi / 2 L$ and $k_{z}=\pi / L$ for the fundamental mode and its first overtone respectively we require that $\epsilon<2 / \pi$. The period ratio is given by

$\frac{P_{1}}{2 P_{2}}=\left(\frac{1-\frac{\epsilon^{2} \pi^{2}}{4}}{1-\frac{\epsilon^{2} \pi^{2}}{16}}\right)^{1 / 2}, \quad \epsilon \pi<2$.

We can expand (34) for small $\epsilon \pi / 2$ :

$\frac{P_{1}}{2 P_{2}}=1-\frac{3 \pi^{2}}{32} \epsilon^{2}-\frac{21 \pi^{4}}{2048} \epsilon^{4}$.

Table 2 gives values of $\epsilon$ for specific temperatures and loop lengths. As before, $\gamma=5 / 3, R=8.3 \times 10^{3} \mathrm{~m}^{2} \mathrm{~s}^{-2} \mathrm{deg}^{-1}$ and the mean molecular weight $\tilde{\mu}=0.6$. The compressive viscosity coefficient is $v_{0}=10^{-17}$ in mks units. Then, for example, a loop of length $100 \mathrm{Mm}(L=50 \mathrm{Mm})$ with temperature $T_{0}=6 \mathrm{MK}$ gives $\epsilon \approx 0.0954$. Thus $\mathcal{V}=0.150$ for the fundamental mode and $\mathcal{V}=0.300$ for the first overtone.

Table 2 shows that for coronal values of the coefficient of compressive viscosity in the majority of cases remains small and so the period ratio remains close to unity and so we conclude that the effect of compressive viscosity on the period ratio is generally small. However, for very hot short loops compressive viscosity may have a significant effect on the period ratio.
For example, a loop of temperature $8 \mathrm{MK}$ and length $50 \mathrm{Mm}$ gives $\epsilon \approx 0.452$ (corresponding to $\mathcal{V}=0.710$ for the fundamental mode and $\mathcal{V}=1.420$ for the first overtone) which leads to a period ratio $P_{1} / 2 P_{2}=0.753$. However, a loop of the same length but hotter temperature $T_{0}=9 \mathrm{MK}$, for example, gives $\epsilon \approx 0.644$ (corresponding to $\mathcal{V}=1.012$ for the fundamental mode and $V=2.023$ for the first overtone) and now the compressive viscosity prevents the first overtone from occurring since it violates the condition $\epsilon<2 / \pi(\mathcal{V}<2)$. In this case it suggests the first overtone is entirely damped.

\section{Combined effects of thermal conduction and compressive viscosity}

We turn now to consider the combined effects of thermal conduction and compressive viscosity determined by the dispersion relation (15). This cubic may be solved for $\Omega$ using the Cardano and Vièta substitution method as before, giving solution (20) as before (with $\operatorname{real}(\Omega)>0$ selected) and where now

$$
\begin{aligned}
C_{1}= & 36 \mathcal{V}-8 \mathcal{V}^{3}+\left(36 \gamma-108+12 \gamma \mathcal{V}^{2}\right) \mathcal{D} \\
& +12 \gamma^{2} \mathcal{V} \mathcal{D}^{2}-8 \gamma^{3} \mathcal{D}^{3} \\
C_{2}= & -12+3 \mathcal{V}^{2}+\left(54 \mathcal{V}-30 \gamma \mathcal{V}+6 \gamma \mathcal{V}^{3}-12 \mathcal{V}^{3}\right) \mathcal{D} \\
& +\left(54 \gamma+3 \gamma^{2}-81+18 \gamma \mathcal{V}^{2}-24 \gamma^{2} \mathcal{V}^{2}+3 \gamma^{2} \mathcal{V}^{4}\right) \mathcal{D}^{2} \\
& +\left(18 \gamma^{2} \mathcal{V}+6 \gamma^{3} \mathcal{V}-6 \gamma^{3} \mathcal{V}^{3}\right) \mathcal{D}^{3}+\left(3 \gamma^{4} \mathcal{V}^{2}-12 \gamma^{3}\right) \mathcal{D}^{4} \\
C_{3}= & 1-\frac{1}{3} \mathcal{V}^{2}+\frac{1}{3} \gamma \mathcal{V}-\frac{1}{3} \gamma^{2} \mathcal{D}^{2} \\
C_{4}= & \frac{1}{3}(\mathcal{V}+\gamma \mathcal{D})
\end{aligned}
$$

When $\mathcal{V}=0$, expressions (36) reduce to those given in (22).

From the solution for the frequency the period ratio may be formed as before. For each particular loop length and temperature the thermal conduction parameter $d$ and the compressive viscosity parameter $\epsilon$ are uniquely determined as given in Tables 1 and 2, and these in turn give specific values for $\mathcal{D}$ and $\mathcal{V}$. Figures 3 and 4 show the variation of the period ratio $P_{1} / 2 P_{2}$ with loop length $2 L$ and temperature $T_{0}$ respectively. The figures indicate that the period ratio has a tendency to reduce for short hot loops such as those observed by SUMER. As an example, for a loop of length $2 L=100 \mathrm{Mm}$ and temperature $T_{0}=10 \mathrm{MK}$ $\left(\mathcal{D}=9.5795\right.$ and $\mathcal{V}=0.442$ ) the period ratio is $P_{1} / 2 P_{2} \approx 0.487$. For shorter loops than this at such high temperatures the period ratio may not be formed as the first harmonic is damped immediately. By contrast, for TRACE loops, a loop of length $2 L=100 \mathrm{Mm}$ but with temperature $T_{0}=1 \mathrm{MK}(\mathcal{D}=0.0096$ 


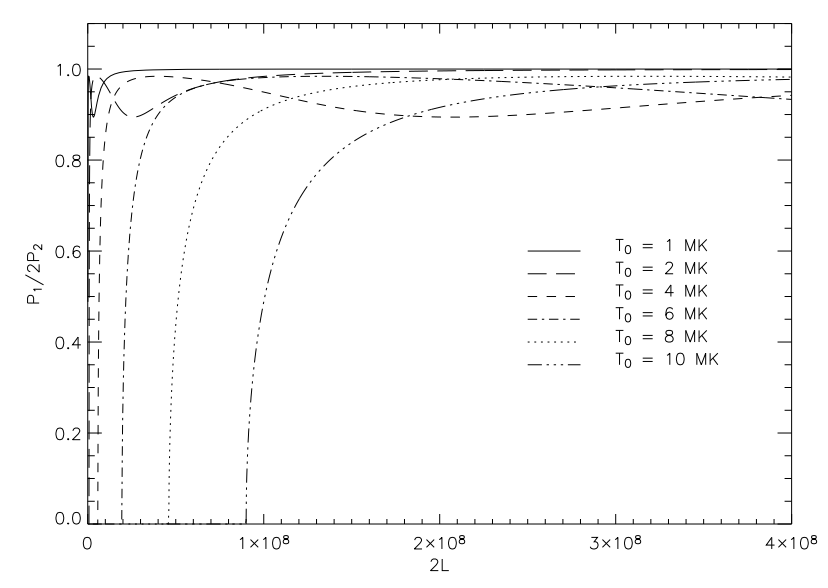

Fig. 3. The period ratio $P_{1} / 2 P_{2}$ as a function of loop length $2 L$ (in metres) for various loop temperatures $T_{0}$.

and $\mathcal{V}=0.000442$ ) produces a period ratio that is very close to unity $\left(P_{1} / 2 P_{2} \approx 1.00\right)$.

As before, it is straightforward to obtain a series expansion for $\Omega$ in powers of $\mathcal{D}$ and $\mathcal{V}$ directly from the dispersion relation (15). Eliminating imaginary terms to derive the real part of the frequency we have

$$
\begin{aligned}
\operatorname{real}(\Omega)= & 1-\frac{1}{8} \mathcal{V}^{2}-\frac{1}{8}(\gamma-1)(\gamma+3) \mathcal{D}^{2}+\frac{1}{4}(\gamma-1) \mathcal{D} \mathcal{V} \\
& -\frac{3}{64}(\gamma-1)(\gamma-5) \mathcal{D}^{2} \mathcal{V}^{2}+\ldots
\end{aligned}
$$

The period ratio for small $\mathcal{D}$ and $\mathcal{V}$ (i.e. small $d$ and $\epsilon$ ) then follows as

$$
\begin{aligned}
\frac{P_{1}}{2 P_{2}}= & 1-\frac{3}{32}(\epsilon \pi)^{2}-\frac{3}{32}(\gamma-1)(\gamma+3)(d \pi)^{2} \\
& +\frac{3}{16}(\gamma-1) d \epsilon \pi^{2}+\ldots ;
\end{aligned}
$$

with $\gamma=5 / 3$ this is

$$
\frac{P_{1}}{2 P_{2}}=1-\frac{3}{32}(\epsilon \pi)^{2}-\frac{7}{24}(d \pi)^{2}+\frac{1}{8} d \epsilon \pi^{2}+\ldots
$$

These formula may be used as a guide when considering Figs. 3 and 4.

Figure 3 indicates that for loops of relatively low temperature (1-2 MK) such as observed by TRACE, the period ratio under the effects of thermal conduction and compressive viscosity hardly deviates from unity. Increasing the temperature increases the parameters $d$ and $\epsilon$, leading to the period ratio decreasing from unity. However, increasing the loop length acts to balance out the effects of the increase in temperature (as $d$ and $\epsilon$ are inversely proportional to $L$ ). Accordingly, in long loops the period ratio hardly departs from unity. For short hot (6-8 MK) loops such as observed by SUMER the period ratio may depart substantially from unity. The overall behaviour, is that for infinitely short loops the period ratio increases from zero to unity before experiencing a localised minimum value which mimics the behaviour of the period ratio for thermal conduction alone, finally the period ratio again tends to unity for long loops. In terms of the thermal conduction and compressive viscosity parameters, $d$ and $\epsilon$, the period ratio behaves in a manner indicated by the approximate result (39).

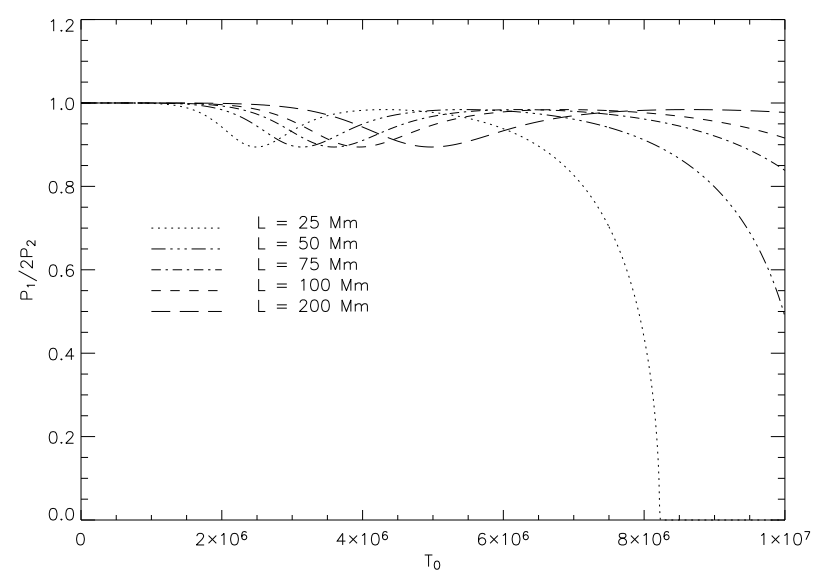

Fig. 4. The period ratio $P_{1} / 2 P_{2}$ as a function of temperture $T_{0}\left({ }^{\circ} \mathrm{K}\right)$ for various half-loop lengths $L$.

Figure 4 gives further information about the period ratio in relation to the loop temperature and length. For all loop lengths considered a low temperature produces small values of $d$ and $\epsilon$ and the period ratio is close to unity (as noted from Fig. 3). For high temperature loops a short loop length can cause the period ratio to depart from unity. Interestingly, Fig. 4 shows that for each loop length the period ratio exhibits a dip as it first decreases from unity and then increases, before again decreasing at high temperature. The dip occurs at relatively low loop temperatures for short loop lengths. This is likely to be associated with the fact that the period ratio under thermal conduction alone has a minimum, as shown in Fig. 1. This may indicate that thermal conduction dominates the period ratio at low temperatures until at a high enough temperature (prescribed individually for each loop length) compressive viscosity dominates and causes the period ratio to fall substantially from unity.

\section{Discussion and conclusions}

We have considered the effects of both thermal conduction and compressive viscosity on the period ratio $P_{1} / 2 P_{2}$. Typically, for both TRACE and SUMER loops thermal conduction has a negligible effect on the period ratio in that it does not substantially reduce the period ratio from unity. For TRACE loops compressive viscosity is also unlikely to be important. However, for short hot SUMER loops compressive viscosity may become important and for such loops a reduction of the period ratio to as little as $P_{1} / 2 P_{2}=0.753$, for example, is possible. This effect is in keeping with the recent observations of Srivastava \& Dwivedi (2010). In fact, under the effect of compressive viscosity, the period ratio is zero in the limiting case since in some cases compressive viscosity may have more of a marked effect on the first overtone, damping it immediately; consequently, the period ratio may not even be formed.

It is of interest to compare the frequency shifts arising from damping effects with those occurring due to stratification. McEwan et al. (2006) show that in an isothermal atmosphere stratified under gravity the period ratio is determined by

$$
\frac{P_{1}}{2 P_{2}}=\left[\frac{1+\left(\frac{L}{2 \pi \Lambda_{c}}\right)^{2}}{1+\left(\frac{L}{\pi \Lambda_{c}}\right)^{2}}\right]^{1 / 2}
$$


where $\Lambda_{c}=p_{0} / g \rho_{0}=c_{\mathrm{s}}^{2} / \gamma g$ is the pressure scale height of the atmosphere and $g$ is the gravitational acceleration. Equation (40) shows that the period ratio declines from a value of unity in short $\left(L \ll \Lambda_{c}\right)$ loops to 0.5 in very long $\left(L \gg \Lambda_{c}\right)$ loops. For loops that are comparable in size to the pressure scale height $\Lambda_{c}$, so that $2 L \approx \Lambda_{c}$, Eq. (40) gives a period ratio of 0.99 . Thus only in very long loops is the effect of gravity important in an isothermal atmosphere.

However, the effect of stratification on the sound speed is important, just as noted for kink modes and the kink speed (Andries et al. 2005a,b; McEwan et al. 2006, 2008). We can deduce frequency shifts for sound waves from the work of McEwan et al. (2006, 2008). Figure 6 in McEwan et al. (2006) shows that in a non-isothermal atmosphere a period ratio of about 0.8 is to be expected for loops of length $2 L=\Lambda_{c}$, with stronger shifts from unity in longer loops (e.g. $P_{1} / 2 P_{2} \approx 0.7$ for $2 L=4 \Lambda_{c}$.)

We conclude that damping by thermal conduction does not play a key role in the departure of $P_{1} / 2 P_{2}$ from unity. For TRACE loops we conclude that compressive viscosity is also negligible. As such, longitudinal density structuring remains the dominant effect (Andries et al. 2005a,b; McEwan et al. 2006, 2008; Verth \& Erdélyi 2008). For SUMER loops, on the other hand, it may be the case that compressive viscosity plays a role in the reduction of the period ratio from unity. In general, though, damping effects such as caused by thermal conduction or compressive viscosity do not bring about significant shifts in the period ratio $P_{1} / 2 P_{2}$ from unity. It would seem that longitudinal structuring is the most likely cause of a period ratio shift below unity, though the combined effect of longitudinal structuring and damping may prove to be of interest.

Acknowledgements. C.K.M. acknowledges financial support from the Carnegie Trust. Discussions with Dr. I. De Moortel and Prof. A. W. Hood are gratefully acknowledged.

\section{References}

Abramowitz, M., \& Stegun, I. A. 1965, Handbook of Mathematical Functions Andries, J., Arregui, I., \& Goossens, M. 2005a, ApJ, 624, L57

Andries, J., Goossens, M., Hollweg, J. V., Arregui, I., \& Van Doorsselaere, T. 2005b, A\&A, 430, 1109

Andries, J., van Doorsselaere, T., Roberts, B., et al. 2009, Space Sci. Rev., 149, 3

Aschwanden, M. J., Fletcher, L., Schrijver, C. J., \& Alexander, D. 1999, ApJ, 520,880

Aschwanden, M. J., de Pontieu, B., Schrijver, C. J., \& Title, A. M. 2002, Sol. Phys., 206, 99

De Moortel, I., \& Brady, C. S. 2007, ApJ, 664, 1210

De Moortel, I., \& Hood, A. W. 2003, A\&A, 408, 755

De Moortel, I., Ireland, J., \& Walsh, R. W. 2000, A\&A, 355, L23

De Moortel, I., Ireland, J., Hood, A. W., \& Walsh, R. W. 2002a, A\&A, 387, L13
De Moortel, I., Ireland, J., Walsh, R. W., \& Hood, A. W. 2002b, Sol. Phys., 209, 61

DeForest, C. E., \& Gurman, J. B. 1998, ApJ, 501, L217

Díaz, A. J., Donnelly, G. R., \& Roberts, B. 2007, A\&A, 476, 359

Donnelly, G. R., Díaz, A. J., \& Roberts, B. 2006, A\&A, 457, 707

Dymova, M. V., \& Ruderman, M. S. 2007, A\&A, 463, 759

Erdélyi, R., \& Morton, R. J. 2009, A\&A, 494, 295

Field, G. B. 1965, ApJ, 142, 531

Goossens, M., Andries, J., \& Arregui, I. 2006, Phil. Trans. R. Soc. A, 364, 433

Hollweg, J. V. 1985, J. Geophys. Res., 90, 7620

Inglis, A. R., Van Doorsselaere, T., Brady, C. S., \& Nakariakov, V. M. 2009, A\&A, 503, 569

Marsh, M. S., Walsh, R. W., \& Plunkett, S. 2009, ApJ, 697, 1674

McEwan, M. P., \& De Moortel, I. 2006, A\&A, 448, 763

McEwan, M. P., Donnelly, G. R., Díaz, A. J., \& Roberts, B. 2006, A\&A, 460, 893

McEwan, M. P., Díaz, A. J., \& Roberts, B. 2008, A\&A, 481, 819

Melnikov, V. F., Reznikova, V. E., Shibasaki, K., \& Nakariakov, V. M. 2005, A\&A, 439, 727

Mendoza-Briceño, C. A., Erdélyi, R., \& Sigalotti, L. D. G. 2004, ApJ, 605, 493

Morton, R. J., \& Erdélyi, R. 2009, A\&A, 502, 315

Nakariakov, V. M., \& Verwichte, E. 2005, Living Rev. Sol. Phys., 2, 3

Nakariakov, V. M., Ofman, L., Deluca, E. E., Roberts, B., \& Davila, J. M. 1999, Science, 285,862

Nakariakov, V. M., Melnikov, V. F., \& Reznikova, V. E. 2003, A\&A, 412, L7

Ofman, L., \& Wang, T. 2002, ApJ, 580, L85

Ofman, L., Davila, J. M., \& Steinolfson, R. S. 1994, ApJ, 421, 360

Ofman, L., Romoli, M., Poletto, G., Noci, G., \& Kohl, J. L. 1997, ApJ, 491, L111

Ofman, L., Nakariakov, V. M., \& DeForest, C. E. 1999, ApJ, 514, 441

O'Shea, E., Srivastava, A. K., Doyle, J. G., \& Banerjee, D. 2007, A\&A, 473, L13

Pascoe, D. J., Nakariakov, V. M., \& Arber, T. D. 2007, A\&A, 461, 1149

Pascoe, D. J., Nakariakov, V. M., Arber, T. D., \& Murawski, K. 2009, A\&A, 494, 1119

Press, W. H., Flannery, B. P., \& Teukolsky, S. A. 1986, Numerical Recipes: The Art of Scientific Computing (Cambridge: University Press)

Robbrecht, E., Verwichte, E., Berghmans, D., et al. 2001, A\&A, 370, 591

Roberts, B. 2006, Phil. Trans. R. Soc. A, 364, 447

Roberts, B. 2008, in IAU Symp. 247, ed. R. Erdélyi \& C. A. Mendoza-Briceño, 3

Roberts, B., Edwin, P. M., \& Benz, A. O. 1984, ApJ, 279, 857

Ruderman, M. S., \& Roberts, B. 2002, ApJ, 577, 475

Ruderman, M. S., Verth, G., \& Erdélyi, R. 2008, ApJ, 686, 694

Sigalotti, L. D. G., Mendoza-Briceño, C. A., \& Luna-Cardozo, M. 2007, Sol. Phys., 246, 187

Spitzer, L. 1962, Physics of Fully Ionized Gases (New York: Wiley Interscience 2nd edn.)

Srivastava, A. K., \& Dwivedi, B. N. 2010, New Astron., 15, 8

Srivastava, A. K., Zaqarashvili, T. V., Uddin, W., Dwivedi, B. N., \& Kumar, P. 2008, MNRAS, 388, 1899

Van Doorsselaere, T., Nakariakov, V. M., \& Verwichte, E. 2007, A\&A, 473, 959

Verth, G., \& Erdélyi, R. 2008, A\&A, 486, 1015

Verwichte, E., Nakariakov, V. M., Ofman, L., \& Deluca, E. E. 2004, Solar Phys., 223, 77

Wang, T., Solanki, S. K., Curdt, W., Innes, D. E., \& Dammasch, I. E. 2002, ApJ, 574, L101

Wang, T. J., \& Solanki, S. K. 2004, A\&A, 421, L33

Wang, T. J., Solanki, S. K., Innes, D. E., Curdt, W., \& Marsch, E. 2003, A\&A, 402, L17

Wang, T. J., Ofman, L., Davila, J. M., \& Mariska, J. T. 2009, A\&A, 503, L25 